\title{
ASYMPTOTIC BASELINE OF THE HAZARD RATE FUNCTION OF MIXTURES
}

\author{
YULIN LI,* University of Toledo
}

\begin{abstract}
In this article, we consider the limit behavior of the hazard rate function of mixture distributions, assuming knowledge of the behavior of each individual distribution. We show that the asymptotic baseline function of the hazard rate function is preserved under mixture.
\end{abstract}

Keywords: Hazard rate function; mixture; asymptotic behavior

2000 Mathematics Subject Classification: Primary 62E10, 60E15

\section{Introduction}

In recent years, properties of distributions that are preserved under mixture have attracted great interest. For example, Lynch (1999) found conditions under which the increasing failure rate (IFR) class is preserved. Block et al. (2003b) and Li (2005) explored conditions for the preservation of some other classes. As for the preservation of the shape of mixture distributions, Block and Joe (1997) and Block et al. (2003a) studied their initial and tail behaviors, especially their increasing and decreasing behaviors. Savits (2003) studied the preservation of distributions with generalized bathtub-shaped hazard rate functions.

In the study of the preservation of tail behavior properties of the mixture distribution, there are several approaches. Block and Joe (1997) considered the eventual increasing or decreasing behavior. Finkelstein (2001) discussed the difference between the hazard rate of the mixture and those of the strongest subpopulations. Block et al. (1993) studied the limit of the hazard rate of the mixture when each individual hazard rate limit exists.

In this paper, instead of assuming that each individual hazard rate has a limit, we assume that there exists an asymptotic baseline function such that the ratio of each individual hazard rate function to this asymptotic baseline function has a limit. We show that, under certain conditions, the ratio of the hazard rate function of the mixture to the asymptotic baseline function has a limit. As in the case of Block et al. (1993), the limit is shown to be the essential infimum. We also show that this limit, if it exists, should always be the essential infimum if the convergence of the ratio is uniform. As an easy corollary of the above results, we find that the ratio of the hazard function of the mixture to a similar integration of the asymptotic baseline function also converges to the essential infimum.

Received 8 September 2004; revision received 14 December 2004.

* Postal address: Department of Mathematics, University of Toledo, Toledo, OH 43606, USA. Email address: li.yulin@utoledo.edu 


\section{Main result}

\subsection{The conditions for existence}

Assume that there is a family of life distributions with densities $\left\{f_{\omega}, \omega \in \Omega\right\}$ and a probability space $(\Omega, \mathcal{F}, \mathrm{P})$, with $f_{\omega}(t)$ being a measurable function of $(\omega, t)$. We consider the mixture

$$
f(t)=\int_{\Omega} f_{\omega}(t) \mathrm{P}(\mathrm{d} \omega) .
$$

Denote the survival function by $\bar{F}$. Then, the hazard rate function of the mixture distribution is

$$
r(t)=\frac{f(t)}{\bar{F}(t)},
$$

while, for an individual distribution with survival function $\bar{F}_{\omega}(t)$, the hazard rate function is

$$
r_{\omega}(t)=\frac{f_{\omega}(t)}{\bar{F}_{\omega}(t)} .
$$

Suppose that there is a positive, continuous function $\rho(t)$ satisfying

$$
\int_{0}^{t} \rho(s) \mathrm{d} s \rightarrow \infty
$$

as $t \rightarrow \infty$, such that $r_{\omega}(t) / \rho(t) \rightarrow \lambda_{\omega}$ uniformly for $\omega \in \Omega$ as $t \rightarrow \infty$. Let $\lambda^{*}$ denote the essential infimum of $\lambda_{\omega}$ on $\Omega$ with respect to $\mathrm{P}$. That is, $\mathrm{P}\left\{\omega: \lambda_{\omega}<\lambda^{*}\right\}=0$ and, for any $\varepsilon>0, \mathrm{P}\left\{\omega: \lambda_{\omega}<\lambda^{*}+\varepsilon\right\}>0$ or, simply,

$$
\underset{\omega \in \Omega}{\operatorname{essinf}} \lambda_{\omega}=\inf \left\{0 \leq \lambda<\infty: \mathrm{P}\left\{\omega \in \Omega: \lambda_{\omega} \leq \lambda\right\}>0\right\} .
$$

In the case that $\rho(t) \equiv 1$ or, equivalently, the limit of the hazard rate function exists and the convergence is uniform, Block et al. (1993) gave the following result about the hazard rate function of the mixture.

Theorem 2.1. Consider the mixture failure rate as given in (2.2). Assume that

(i) $r_{\omega}(t)$ converges to $\lambda_{\omega}$ uniformly on $\Omega$ as $t \rightarrow \infty$, where $0 \leq \lambda_{\omega} \leq \infty$.

Let $I=\left\{\omega \in \Omega: \lambda_{\omega}=\infty\right\}$. If $0<\mathrm{P}(I)<1$, further assume that

(ii) there exist $L$ and $T, 0 \leq L, T<\infty$, such that $r_{\omega}(t) \leq \exp (L t)$ for all $\omega \in I$ and all $t \geq T$.

Then

$$
\lim _{t \rightarrow \infty} r(t)=\lambda^{*}=\underset{\omega \in \Omega}{\operatorname{essinf}} \lambda_{\omega}
$$

Remark 2.1. (I) If $\mathrm{P}(I)=0$ or $\mathrm{P}(I)=1$, condition (ii) is not needed.

(II) Theorem 2.1 can be interpreted as saying that the hazard rate of the mixture converges to the limit of hazard rate of the strongest subpopulation. 
It is easy to show that if the uniform convergence assumption is not satisfied, then the limit may not exist. Block et al. (2003a) gave an example showing that the limit may not exist if the exponential growth condition (ii) is not satisfied. The following example is helpful to motivate the extension of Theorem 2.1 .

Example 2.1. (Gupta and Gupta (1996).) Let $r_{0}(t)=\gamma t^{\gamma-1}$, with $\gamma>1$, be an IFR Weibull baseline hazard rate and set $r(t, \omega)=\omega r_{0}(t)$. Consider a gamma mixture $\mathrm{P}$ on $\Omega=(0, \infty)$ :

$$
\mathrm{P}(\mathrm{d} \omega)=\frac{1}{\beta^{\lambda} \Gamma(\lambda)} \omega^{\lambda-1} \exp (-\omega / \beta) \mathrm{d} \omega,
$$

for some $\beta>0$ and $\lambda>0$. It is easy to see that $\lambda_{\omega}=\lim _{t \rightarrow \infty} r(t, \omega)=\infty$ for every $\omega \in \Omega$. Hence, $\lambda^{*}=\infty$. Condition (i) of Theorem 2.1 is violated in that the convergence is not uniform. We can explicitly calculate $r(t)$ to be

$$
r(t)=\frac{\lambda \beta \gamma t^{\gamma-1}}{1+\beta t^{\gamma}}
$$

which converges to 0 as $t \rightarrow \infty$.

However, if we take $\rho(t)=r_{0}(t)$ then, as $t \rightarrow \infty$,

$$
\frac{r_{\omega}(t)}{\rho(t)}=\omega
$$

and, hence, $r_{\omega}(t) / \rho(t) \rightarrow \omega$ uniformly for $\omega \in \Omega$. That is, this family has a baseline hazard rate here. To deal with situations like this, we have the following result.

Theorem 2.2. Consider the mixture hazard rate as given in (2.2). Assume that

(i) $r_{\omega}(t) / \rho(t)$ converges to $\lambda_{\omega}$ uniformly on $\Omega$ as $t \rightarrow \infty$, where $0 \leq \lambda_{\omega} \leq \infty$.

Let $I=\left\{\omega \in \Omega: \lambda_{\omega}=\infty\right\}$. If $0<\mathrm{P}(I)<1$, further assume that

(ii) there exist $L$ and $T, 0 \leq L, T<\infty$, such that $r_{\omega}(t) \leq \rho(t) \exp \left(L \int_{0}^{t} \rho(s) \mathrm{d} s\right)$ for all $\omega \in I$ and all $t \geq T$.

Then

$$
\begin{aligned}
\lim _{t \rightarrow \infty} \frac{r(t)}{\rho(t)} & =\lim _{t \rightarrow \infty} \frac{1}{\rho(t)} \frac{\int_{\Omega} f_{\omega}(t) \mathrm{P}(\mathrm{d} \omega)}{\int_{\Omega} \bar{F}_{\omega}(t) \mathrm{P}(\mathrm{d} \omega)} \\
& =\lambda^{*} \\
& =\underset{\omega \in \Omega}{\operatorname{essinf}} \lambda_{\omega} .
\end{aligned}
$$

Remark 2.2. The result of Block et al. (1993), as stated in Theorem 2.1 here, is a special case of Theorem 2.2: it corresponds to the case $\rho(t)=1$. In the first proof of Theorem 2.2, we show that it follows from Theorem 2.1 by a time-scale change. Note that we do not need to assume that $\rho(t)$ is monotone.

Proof of Theorem 2.2. Consider the time change $s=\int_{0}^{t} \rho(u) \mathrm{d} u$. Let $t \equiv t(s)$ denote the inverse function. Set $\bar{G}(s)=\bar{F}(t(s))$ and $\bar{G}(s \mid \omega)=\bar{F}(t(s) \mid \omega)$ for $s \in \mathbb{R}^{+}$and $\omega \in \Omega$. Then the density of $\bar{G}$ is

$$
g(s)=f(t(s)) t^{\prime}(s)=\frac{f(t(s))}{\rho(t(s))},
$$


where a prime denotes differentiation. Similarly, the density of $\bar{G}(s \mid \omega)$ is

$$
g(s \mid \omega)=f(t(s) \mid \omega) t^{\prime}(s)=\frac{f(t(s) \mid \omega)}{\rho(t(s))} .
$$

Clearly, the hazard rate $r(s \mid \omega)$ of $\bar{G}(s \mid \omega)$ satisfies

$$
\begin{aligned}
r(s \mid \omega) & =\frac{g(s \mid \omega)}{\bar{G}(s \mid \omega)} \\
& =\frac{f(t(s) \mid \omega) / \rho(t(s))}{\bar{G}(s \mid \omega)} \\
& =\frac{r(t(s) \mid \omega)}{\rho(t(s))} .
\end{aligned}
$$

Therefore, we find that $r(s \mid \omega) \rightarrow \lambda_{\omega}$ and $r(s \mid \omega) \leq \exp (L s)$. Hence, as $s \rightarrow \infty$,

$$
\begin{aligned}
\frac{r(t(s))}{\rho(t(s))} & =\frac{\int_{\Omega} f(t(s) \mid \omega) \mathrm{P}(\mathrm{d} \omega)}{\int_{\Omega} \bar{F}(t(s) \mid \omega) \mathrm{P}(\mathrm{d} \omega)} \frac{1}{\rho(t(s))} \\
& =\frac{\int_{\Omega} g(s \mid \omega) \mathrm{P}(\mathrm{d} \omega)}{\int_{\Omega} \bar{G}(s \mid \omega) \mathrm{P}(\mathrm{d} \omega)} \\
& =\frac{g(s)}{\bar{G}(s)} \\
& \rightarrow \lambda^{*},
\end{aligned}
$$

where the last step follows from Theorem 2.1. Hence, $r(t) / \rho(t) \rightarrow \lambda^{*}$ as $t \rightarrow \infty$.

Using this result, we can obtain a parallel result about the limiting behavior of the hazard function of the mixture distribution.

Corollary 2.1. Let $R(t)=\int_{0}^{t} r(s) \mathrm{d} s$ denote the hazard function of the mixture distribution specified in (2.1), and let $P(t)=\int_{0}^{t} \rho(s) \mathrm{d}$ s. Under the conditions of Theorem 2.2, as $t \rightarrow \infty$, we have $R(t) / P(t) \rightarrow \lambda^{*}$.

Proof. If $r(t)$ is a continuous function, this follows directly from the result of Theorem 2.2, by applying l'Hôpital's rule. Otherwise, it can be proved in a way similar to the proof of l'Hôpital's rule.

Although Block et al. (2003a) have given an example in which, if the exponential growth condition is not satisfied, the limit may not exist, it is still possible to find some other conditions, not concerning growth rate, under which the limit is the essential infimum. In the following, we outline another proof of Theorem 2.2 that is almost exactly the same as that given by Block et al. (1993) for Theorem 2.1. A close examination of the proof will give other conditions under which the limit exists. As in Block et al. (1993), we need three lemmas. Their proofs are similar to their corresponding lemmas in Block et al. (1993) and, therefore, are omitted here.

Lemma 2.1. Assume that $\lambda^{*}<\infty$. Then, given any $\lambda_{0}>\lambda^{*}$, there exists a constant $c>0$ such that

$$
\int \bar{F}_{\omega}(t) \mathrm{P}(\mathrm{d} \omega) \geq c \exp \left(-\lambda_{0} \int_{0}^{t} \rho(s) \mathrm{d} s\right)
$$

for all sufficiently large $t$. 
Lemma 2.2. If there exist nonnegative constants $L$ and $T$ such that, for all $t \geq T$, we have $r_{\omega}(t) \leq \rho(t) \exp \left(L \int_{0}^{t} \rho(s) \mathrm{d} s\right)$ on the set $I=\left\{\omega: \lambda_{\omega}=\infty\right\}$, then, for any $\gamma>0$, as $t \rightarrow \infty$ we have

$$
\frac{1}{\rho(t)} \exp \left(\gamma \int_{0}^{t} \rho(s) \mathrm{d} s\right) \int_{I} r_{\omega}(t) \bar{F}_{\omega}(t) \mathrm{P}(\mathrm{d} \omega) \rightarrow 0 .
$$

Lemma 2.3. If $\lambda_{0}>\lambda^{*}$ is given, there exist $\lambda_{1}>\lambda^{*}$ and $C>0$ such that, for sufficiently large $t$,

$$
\int_{B} \lambda_{\omega} \bar{F}_{\omega}(t) \mathrm{P}(\mathrm{d} \omega) \leq C \exp \left(-\lambda_{1} \int_{0}^{t} \rho(s) \mathrm{d} s\right),
$$

where $B=\left\{\omega \in \Omega: \lambda_{0}<\lambda_{\omega}<\infty\right\}$.

We are now ready to give the second proof of Theorem 2.2. It is similar to the proof of Theorem 2.1, which is given in Block et al. (1993).

Proof of Theorem 2.2. First, we introduce a probability measure $M_{t}$ on $\Omega$ via its RadonNikodým derivative

$$
\frac{\mathrm{d} M_{t}}{\mathrm{~d} P}\left(\omega_{0}\right)=\frac{\bar{F}_{\omega_{0}}(t)}{\int \bar{F}_{\omega}(t) \mathrm{P}(\mathrm{d} \omega)}, \quad \omega_{0} \in \Omega .
$$

Then

$$
\frac{r(t)}{\rho(t)}=\int \frac{r_{\omega}(t)}{\rho(t)} M_{t}(\mathrm{~d} \omega) .
$$

Suppose that $\lambda^{*}=\infty$ : the result then follows from the assumption of uniform convergence since, in this case, $\lambda_{\omega}=\infty$ for all $\omega \in \Omega$. Now consider the case in which $0 \leq \lambda^{*}<\infty$. In this case, we have

$$
\begin{aligned}
\left|\int \frac{r_{\omega}(t)}{\rho(t)} M_{t}(\mathrm{~d} \omega)-\lambda^{*}\right| \leq & \int_{I}\left|\frac{r_{\omega}(t)}{\rho(t)}-\lambda^{*}\right| M_{t}(\mathrm{~d} \omega)+\int_{I^{\mathrm{c}}}\left|\frac{r_{\omega}(t)}{\rho(t)}-\lambda_{\omega}\right| M_{t}(\mathrm{~d} \omega) \\
& +\int_{I^{\mathrm{c}}}\left|\lambda_{\omega}-\lambda^{*}\right| M_{t}(\mathrm{~d} \omega) .
\end{aligned}
$$

We will show that each of the three terms on the right-hand side of (2.3) tends to 0 . Since $r_{\omega}(t) / \rho(t) \rightarrow \infty$ uniformly on $I$ as $t \rightarrow \infty$, there exists a constant $K$ such that $\left|r_{\omega}(t) / \rho(t)-\lambda^{*}\right| \leq K r_{\omega}(t) / \rho(t)$ for all $\omega \in I$ and $t$ sufficiently large. Hence, as $t \rightarrow \infty$, the first term on the right-hand side of (2.3) goes to 0 since, by Lemmas 2.1 and 2.2,

$$
\begin{aligned}
\int_{I}\left|\frac{r_{\omega}(t)}{\rho(t)}-\lambda^{*}\right| M_{t}(\mathrm{~d} \omega) & \leq \frac{K}{\rho(t)} \frac{\int_{I} r_{\omega}(t) \bar{F}_{\omega}(t) \mathrm{P}(\mathrm{d} \omega)}{\int \bar{F}_{\omega}(t) \mathrm{P}(\mathrm{d} \omega)} \\
& \leq \frac{K}{C \rho(t)} \exp \left(\eta \int_{0}^{t} \rho(s) \mathrm{d} s\right) \int_{I} r_{\omega}(t) \bar{F}_{\omega}(t) \mathrm{P}(\mathrm{d} \omega) \\
& \rightarrow 0
\end{aligned}
$$

The convergence to 0 of the second term on the right-hand side of (2.3), as $t \rightarrow \infty$, follows from the uniform convergence on $I^{\mathrm{c}}$ of $r_{\omega}(t) / \rho(t)$ to $\lambda_{\omega}$. For the third term, let $\varepsilon>0$, choose $\lambda_{0} \in\left(\lambda^{*}, \lambda^{*}+\varepsilon\right)$, and let $B=\left\{\omega \in \Omega: \lambda_{0}<\lambda_{\omega}<\infty\right\}$. Then we have

$$
\int_{I^{\mathrm{c}}}\left|\lambda_{\omega}-\lambda^{*}\right| M_{t}(\mathrm{~d} \omega) \leq \varepsilon+\int_{B}\left|\lambda_{\omega}-\lambda^{*}\right| M_{t}(\mathrm{~d} \omega) .
$$


From Lemma 2.3, there exist a $\lambda_{1}>\lambda^{*}$ and a constant $C_{1}>0$ such that, for $t$ sufficiently large,

$$
\int_{B} \lambda_{\omega} \bar{F}_{\omega}(t) \mathrm{P}(\mathrm{d} \omega) \leq C_{1} \exp \left(-\lambda_{1} \int_{0}^{t} \rho(s) \mathrm{d} s\right) .
$$

From Lemma 2.1, we conclude that, for sufficiently large $t$,

$$
\begin{aligned}
\int_{B}\left|\lambda_{\omega}-\lambda^{*}\right| M_{t}(\mathrm{~d} \omega) & \leq \frac{\int_{B} \lambda_{\omega} \bar{F}_{\omega}(t) \mathrm{P}(\mathrm{d} \omega)}{\int \bar{F}_{\omega}(t) \mathrm{P}(\mathrm{d} \omega)} \\
& \leq \frac{C_{1}}{C} \exp \left(\left(\lambda_{0}^{\prime}-\lambda_{1}\right) \int_{0}^{t} \rho(s) \mathrm{d} s\right),
\end{aligned}
$$

where $\lambda_{0}^{\prime}=\frac{1}{2}\left(\lambda_{1}+\lambda^{*}\right)$. Therefore, we have

$$
\limsup _{t \rightarrow \infty} \int_{I^{\mathrm{c}}}\left|\lambda_{\omega}-\lambda^{*}\right| M_{t}(\mathrm{~d} \omega) \leq \varepsilon
$$

This completes the proof.

From the above proof, we see that the generalized exponential growth condition is only used in Lemma 2.2. Therefore, we can replace the generalized exponential growth condition by the result of Lemma 2.2, or any other condition that ensures the result of Lemma 2.2.

Theorem 2.3. Suppose that, for any $\gamma>0$,

$$
\frac{1}{\rho(t)} \exp \left(\gamma \int_{0}^{t} \rho(s) \mathrm{d} s\right) \int_{I} r_{\omega}(t) \bar{F}_{\omega}(t) \mathrm{P}(\mathrm{d} \omega) \rightarrow 0 \text { as } t \rightarrow \infty .
$$

Then the hazard rate $r(t)$ of the mixture satisfies

$$
\frac{r(t)}{\rho(t)} \rightarrow \lambda^{*} \text { as } t \rightarrow \infty
$$

Another possible variant is as follows.

Condition 2.1. Let $\rho(t)$ be increasing. Assume that there exists a nonnegative constant $T$ such that $f_{\omega}(t)$ is decreasing for all $t \geq T$ on the set $I=\left\{\omega: \lambda_{\omega}=\infty\right\}$.

Theorem 2.4. Suppose that Condition 2.1 holds. Then the hazard rate $r(t)$ of the mixture satisfies

$$
\frac{r(t)}{\rho(t)} \rightarrow \lambda^{*} \text { as } t \rightarrow \infty
$$

Remark 2.3. If $\rho(t) \equiv 1$, we only require that $f_{\omega}(t)$ be decreasing for all $t \geq T$ on the set $I$ for some $T>0$.

In fact, under Condition 2.1, we have the result of Lemma 2.2.

Lemma 2.4. Assume that Condition 2.1 holds. Then, for any $\gamma>0$,

$$
\frac{1}{\rho(t)} \exp \left(\gamma \int_{0}^{t} \rho(s) \mathrm{d} s\right) \int_{I} r_{\omega}(t) \bar{F}_{\omega}(t) \mathrm{P}(\mathrm{d} \omega) \rightarrow 0 \text { as } t \rightarrow \infty,
$$


Proof. On the set $I$, as $t \rightarrow \infty$,

$$
r_{\omega}(t) / \rho(t) \rightarrow \infty
$$

uniformly; hence, by l'Hôpital's rule,

$$
\frac{\ln \bar{F}_{\omega}(t)}{\int_{0}^{t} \rho(s) \mathrm{d} s} \rightarrow-\infty
$$

Therefore, for $K>\gamma$, there exists a $T_{1}>T$ such that

$$
\bar{F}_{\omega}(t)<\exp \left(-K \int_{0}^{t} \rho(s) \mathrm{d} s\right) \text { for any } t>T_{1} .
$$

Thus, we have

$$
\begin{aligned}
\frac{1}{\rho(t)} & \exp \left(\gamma \int_{0}^{t} \rho(s) \mathrm{d} s\right) \int_{I} r_{\omega}(t) \bar{F}_{\omega}(t) \mathrm{P}(\mathrm{d} \omega) \\
& \leq \frac{1}{\rho(t)} \exp \left(\gamma \int_{0}^{t} \rho(s) \mathrm{d} s\right) \int_{I} f_{\omega}(t) \mathrm{P}(\mathrm{d} \omega) \\
& \leq \frac{1}{\rho(t)} \exp \left(\gamma \int_{0}^{t} \rho(s) \mathrm{d} s\right) \int_{I} \rho(t) \int_{t-1 / \rho(t)}^{t} f_{\omega}(s) \mathrm{d} s \mathrm{P}(\mathrm{d} \omega) \\
& \leq \frac{1}{\rho(t)} \exp \left(\gamma \int_{0}^{t} \rho(s) \mathrm{d} s\right) \int_{I} \rho(t) \bar{F}_{\omega}\left(t-\frac{1}{\rho(t)}\right) \mathrm{P}(\mathrm{d} \omega) \\
& =\exp \left(\gamma \int_{0}^{t} \rho(s) \mathrm{d} s\right) \int_{I} \bar{F}_{\omega}\left(t-\frac{1}{\rho(t)}\right) \mathrm{P}(\mathrm{d} \omega) \\
& \leq \exp \left(\gamma \int_{0}^{t} \rho(s) \mathrm{d} s\right) \exp \left(-K \int_{0}^{t} \rho(s) \mathrm{d} s\right) \exp \left(K \int_{t-1 / \rho(t)}^{t} \rho(s) \mathrm{d} s\right) \\
& \leq \exp \left(-(K-\gamma) \int_{0}^{t} \rho(s) \mathrm{d} s\right) \exp (K) \\
& \rightarrow 0
\end{aligned}
$$

The increasing condition on $\rho(t)$ can be replaced by another condition.

Theorem 2.5. If $T<\infty$ is such that, for any $\omega_{0} \in I, f_{\omega_{0}}$ is decreasing on $[T, \infty)$, and if $\rho(t) \sim c / t^{\alpha}$ with $\alpha<1$ and $c>0$, then the hazard rate $r(t)$ of the mixture satisfies

$$
\frac{r(t)}{\rho(t)} \rightarrow \lambda^{*} \text { as } t \rightarrow \infty \text {. }
$$

Here, $\rho(t) \sim c / t^{\alpha}$ means that $\lim _{t \rightarrow \infty} \rho(t) /\left(c / t^{\alpha}\right)=1$.

Proof. The proof is similar to the proof of Lemma 2.4.

Remark 2.4. As in Corollary 2.1, we can also show that, under the condition of Theorem 2.4 or the conditions of Theorem 2.5, as $t \rightarrow \infty$ the hazard function of the mixture distribution in (2.1) has an asymptotic baseline function, i.e.

$$
\frac{R(t)}{P(t)} \rightarrow \lambda^{*}
$$




\subsection{The possible value of the limit}

As mentioned in Block et al. (2003a), if the convergence is not uniform then the limit of the hazard rate function may not exist. The following example shows that if the convergence is not uniform but the limit of hazard rate function of the mixture does exist, then the limit could be some number other than the essential infimum. Note that Example 2.1 could also serve as a counterexample.

Example 2.2. Fix $\lambda_{1}$ and $\lambda_{2}, \lambda_{1}>\lambda_{2}>0$. Suppose that we define the following sequence of density functions for $n=1,2, \ldots$ :

$$
f_{n}(t)=\frac{1}{2} \lambda_{1} \mathrm{e}^{-\lambda_{1} t}+\frac{1}{2} \lambda_{2} \mathrm{e}^{-\lambda_{2} t} \frac{1}{\mathrm{e}^{-\lambda_{2}(n-1)}-\mathrm{e}^{-\lambda_{2} n}} \mathbf{1}_{[n-1, n)}(t) .
$$

Here, 1 denotes an indicator function. For $n=1,2, \ldots$, let

$$
p_{n}=\mathrm{e}^{-\lambda_{2}(n-1)}-\mathrm{e}^{-\lambda_{2} n} \text {. }
$$

It is then easy to see that the limit of the hazard rate of $f_{n}$ is $\lambda_{1}$ for all $n$. However, the convergence is not uniform here. Let us consider the mixture with density

$$
f(t)=\sum_{n=1}^{\infty} p_{n} f_{n}(t)
$$

It is not difficult to show that

$$
f(t)=\frac{1}{2} \lambda_{1} \mathrm{e}^{-\lambda_{1} t}+\frac{1}{2} \lambda_{2} \mathrm{e}^{-\lambda_{2} t}
$$

Therefore, from Theorem 2.1, we know that, here, the limit of the hazard rate of the mixture is $\lambda_{2}$, not the essential infimum $\lambda_{1}$.

However, in the case of uniform convergence, Block et al. (2003a) have shown that either the limit does not exist or the limit is the essential infimum in the finite mixture case. Here, we first extend the result to the case in which there is an asymptotic baseline function, and then extend it to the general mixture case.

Lemma 2.5. Suppose that the two hazard rates $r_{1}$ and $r_{2}$ with probability density functions $f_{1}$ and $f_{2}$ and survival functions $\bar{F}_{1}$ and $\bar{F}_{2}$, respectively, satisfy $r_{1}(t) / \rho(t) \rightarrow \lambda_{1}<\infty$ and $r_{2}(t) / \rho(t) \rightarrow \infty$. If the mixture hazard rate

$$
r(t)=\frac{p_{1} f_{1}(t)+p_{2} f_{2}(t)}{p_{1} \bar{F}_{1}(t)+p_{2} \bar{F}_{2}(t)}
$$

satisfies $r(t) / \rho(t) \rightarrow \lambda$ (which is possibly infinite) as $t \rightarrow \infty$, then $\lambda=\lambda_{1}$.

Proof. Note that the survival functions are given by

$$
\bar{F}_{1}(t)=\exp \left(-\int_{0}^{t} r_{1}(s) \mathrm{d} s\right)
$$

and

$$
\bar{F}_{2}(t)=\exp \left(-\int_{0}^{t} r_{2}(s) \mathrm{d} s\right)
$$


Since $\int_{0}^{\infty} \rho(t) \mathrm{d} t=\infty$, it follows that

$$
\frac{\bar{F}_{2}(t)}{\bar{F}_{1}(t)} \rightarrow 0
$$

Therefore,

$$
\frac{p_{1} f_{1}(t) / \rho(t)}{p_{1} \bar{F}_{1}(t)+p_{2} \bar{F}_{2}(t)}=\frac{p_{1}\left(f_{1}(t) / \rho(t)\right) / \bar{F}_{1}(t)}{p_{1}+p_{2} \bar{F}_{2}(t) / \bar{F}_{1}(t)} \rightarrow \frac{p_{1} \lambda_{1}}{p_{1}}=\lambda_{1}
$$

and

$$
\frac{p_{2} f_{2}(t) / \rho(t)}{p_{1} \bar{F}_{1}(t)+p_{2} \bar{F}_{2}(t)} \rightarrow \lambda-\lambda_{1} \geq 0,
$$

with the convention that $\lambda-\lambda_{1}=\infty$ if $\lambda=\infty$. Since

$$
\begin{aligned}
\frac{p_{2} f_{2}(t) / \rho(t)}{p_{1} \bar{F}_{1}(t)} & =\frac{p_{2} f_{2}(t) / \rho(t)}{p_{1} \bar{F}_{1}(t)+p_{2} \bar{F}_{2}(t)} \times \frac{p_{1} \bar{F}_{1}(t)+p_{2} \bar{F}_{2}(t)}{p_{1} \bar{F}_{1}(t)} \\
& \rightarrow \lambda-\lambda_{1},
\end{aligned}
$$

we have

$$
\frac{f_{2}(t) / \rho(t)}{\bar{F}_{1}(t)} \rightarrow \frac{p_{1}}{p_{2}}\left(\lambda-\lambda_{1}\right) .
$$

If $\lambda>\lambda_{1}$ then, since $\ln \bar{F}_{1}(t) / \int_{0}^{t} \rho(s) \mathrm{d} s \rightarrow-\lambda_{1}, f_{2}(t)$ must be greater than

$$
c_{1} \exp \left(-c_{2} \int_{0}^{t} \rho(s) \mathrm{d} s\right) \rho(t)
$$

for some $c_{1}$ and $c_{2}$, and sufficiently large $t$. Were this the case, $\bar{F}_{2}(t)$ would satisfy the following inequality:

$$
\begin{aligned}
\bar{F}_{2}(t) & \geq \int_{t}^{\infty} c_{1} \exp \left(-c_{2} \int_{0}^{s} \rho(u) \mathrm{d} u\right) \rho(s) \mathrm{d} s \\
& =\frac{c_{1}}{c_{2}} \exp \left(-c_{2} \int_{0}^{t} \rho(u) \mathrm{d} u\right) .
\end{aligned}
$$

However, this is impossible. Hence, $\lambda=\lambda_{1}$, as $\lambda \geq \lambda_{1}$.

Next, we generalize the result to the case of a general mixture with an asymptotic baseline function.

Theorem 2.6. Consider the mixture failure rate given in (2.2). Assume that $r_{\omega}(t) / \rho(t)$ converges to $\lambda_{\omega}$ uniformly on $\Omega$ as $t \rightarrow \infty$, where $0 \leq \lambda_{\omega} \leq \infty$. Then either

$$
\lim _{t \rightarrow \infty} r(t) / \rho(t)=\lambda^{*}=\underset{\omega \in \Omega}{\operatorname{ess} \inf } \lambda_{\omega}
$$

or $r(t) / \rho(t)$ does not converge.

Proof. If all the $\lambda_{\omega}$ are finite, or all are infinite, then the result holds by Theorem 2.2. Thus, suppose that some limits are finite and some are infinite, and assume that $r(t) / \rho(t) \rightarrow \xi$, $0 \leq \xi \leq \infty$, as $t \rightarrow \infty$. Set

$$
f(t)=\int_{\Omega} f_{\omega}(t) \mathrm{P}(\mathrm{d} \omega) \text { and } \bar{F}(t)=\int_{\Omega} \bar{F}_{\omega}(t) \mathrm{P}(\mathrm{d} \omega) .
$$


Set $\mathrm{P}_{1}^{*}(A)=\mathrm{P}(A) / \mathrm{P}\left(I^{\mathrm{c}}\right)$ for any measurable set $A \subset I^{\mathrm{c}}$, and $\mathrm{P}_{2}^{*}(A)=\mathrm{P}(A) / \mathrm{P}(I)$ for any measurable set $A \subset I$. Then define

$$
\begin{aligned}
f_{1}^{*}(t) & =\int_{I^{\mathrm{c}}} f_{\omega}(t) \mathrm{P}_{1}^{*}(\mathrm{~d} \omega), \\
\bar{F}_{1}^{*}(t) & =\int_{I^{\mathrm{c}}} \bar{F}_{\omega}(t) \mathrm{P}_{1}^{*}(\mathrm{~d} \omega), \\
f_{2}^{*}(t) & =\int_{I} f_{\omega}(t) \mathrm{P}_{2}^{*}(\mathrm{~d} \omega), \\
\bar{F}_{2}^{*}(t) & =\int_{I} \bar{F}_{\omega}(t) \mathrm{P}_{2}^{*}(\mathrm{~d} \omega) .
\end{aligned}
$$

By Theorem 2.2,

$$
\lim _{t \rightarrow \infty} \frac{f_{1}^{*}(t) / \rho(t)}{\bar{F}_{1}^{*}(t)}=\lambda^{*}
$$

and

$$
\lim _{t \rightarrow \infty} \frac{f_{2}^{*}(t) / \rho(t)}{\bar{F}_{2}^{*}(t)}=\infty .
$$

Since

$$
f(t)=\mathrm{P}\left(I^{\mathrm{c}}\right) f_{1}^{*}(t)+\mathrm{P}(I) f_{2}^{*}(t),
$$

we deduce that $\xi=\lambda^{*}$ from Lemma 2.5 .

\section{Acknowledgements}

The author is grateful to Henry W. Block and Thomas H. Savits for discussions and helpful suggestions on this paper. The author would also like to thank the referee for a careful reading of the manuscript and for pointing out Corollary 2.1. The referee also provided an independent proof of Corollary 2.1 without using the result of Theorem 2.2.

\section{References}

Block, H. W. And Joe, H. (1997). Tail behavior of the failure rate functions of mixtures. Lifetime Data Anal. 3, 269-288.

Block, H. W., LI, Y. AND SAVITs, T. H. (2003a). Initial and final behavior of failure rate functions for mixtures and systems. J. Appl. Prob. 40, 721-740.

Block, H. W., Li, Y. and Savits, T. H. (2003b). Preservation of properties under mixture. Prob. Eng. Inf. Sci. 17, 205-212.

Block, H. W., Mi, J. And SAvits, T. H. (1993). Burn-in and mixed populations. J. Appl. Prob. 30, 692-702.

Finkelstein, M. S. (2001). The failure rate and the mean residual lifetime of mixtures. In System and Bayesian Reliability (Ser. Qual. Reliab. Eng. Statist.), eds Y. Hayakawa, T. Irony and M. Xie. World Scientific, River Edge, NJ.

Gupta, P. L. AND GuPta, R. C. (1996). Ageing characteristics of the Weibull mixtures. Prob. Eng. Inf. Sci. 10, 591-600.

LI, Y. (2005). Preservations of NBUC and NBU(2) classes under mixtures. Prob. Eng. Inf. Sci. 19, 277-288.

LYNCH, J. D. (1999). On conditions for mixtures of increasing failure rate distributions to have an increasing failure rate. Prob. Eng. Inf. Sci. 13, 33-36.

Savits, T. H. (2003). Preservation of generalized bathtub-shaped functions. J. Appl. Prob. 40, 473-484. 